\title{
The prognosis benefits of adjuvant versus salvage radiotherapy for patients after radical prostatectomy with adverse pathological features: a systematic review and meta-analysis
}

Ronggui Tao ${ }^{\dagger}$, Jindong Dai ${ }^{\dagger}$, Yunjin Bai ${ }^{\dagger}$, Jiyu Yang, Guangxi Sun, Xingming Zhang, Jinge Zhao, Hao Zeng and Pengfei Shen*

\begin{abstract}
Background: The appropriate timing of radiotherapy (RT) for patients after radical prostatectomy (RP) with adverse pathological features (APFs) remains controversial. This systematic review was conducted to compare the efficacy of adjuvant radiotherapy (ART) and salvage radiotherapy (SRT).

Methods: PubMed, EMBASE, Web of Science and the Cochrane Library electronic databases were searched to retrieve the required. The hazard ratio (HR) and corresponding 95\% confidence interval (CI) of overall survival (OS), biochemical recurrence-free survival (BRFS) and distant metastases-free survival (DMFS) were extracted. The survival benefits of ART with SRT (including early salvage radiotherapy (ESRT)) were analyzed. The process of the meta-analysis was performed with RevMan version 5.3.

Results: A total of fifteen retrospective studies were finally included in the final analysis including 5586 patients. The pooled analysis indicated that ART could achieve better control of prostate cancer and improve OS $(p=0.0006)$, BRFS $(p<0.0001)$ and DMFS $(p<0.0001)$, when compared to SRT. The subgroup analysis of the 5 -year OS rate demonstrated that the ART group still had survival advantages compared to the SRT group $(p=0.0006)$. However, ART and SRT were comparable in 10-year OS rate $(p=0.07)$. ART had advantages over SRT in both 5 -year $(p=0.0003)$ and 10-year BRFS $(p=0.0003)$. The subgroup analysis with different follow-up starting points from RP or RT was essentially consistent with the above results. The pooled analysis also showed that ART was superior to ESRT on OS $(p=0.008)$ and DMFS ( $p=0.03)$, and comparable to ESRT on BRFS $(p=0.1)$.
\end{abstract}

Conclusions: According to this meta-analysis, ART could be served as a preferential treatment for patients with APFs after RP to improve prognosis. Certainly, high-quality, multicenter randomized controlled trials (RCTs) are expecting to confirm the outcomes of our meta-analysis in the future.

Keywords: Radical prostatectomy, Adverse pathological features, Adjuvant radiotherapy, Salvage radiotherapy, Prognosis

\footnotetext{
* Correspondence: cdhx510@163.com

${ }^{\dagger}$ Ronggui Tao, Jindong Dai and Yunjin Bai are co-first authors and

contributed equally.

Department of Urology, Institute of Urology, and National Clinical Research

Center for Geriatrics, West China Hospital, Sichuan University, No. 37 Guoxue

Xiang, Chengdu 610041, China
}

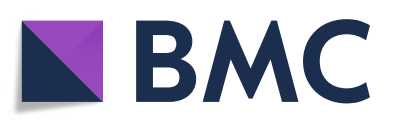

(c) The Author(s). 2019 Open Access This article is distributed under the terms of the Creative Commons Attribution 4.0 International License (http://creativecommons.org/licenses/by/4.0/), which permits unrestricted use, distribution, and reproduction in any medium, provided you give appropriate credit to the original author(s) and the source, provide a link to the Creative Commons license, and indicate if changes were made. The Creative Commons Public Domain Dedication waiver (http://creativecommons.org/publicdomain/zero/1.0/) applies to the data made available in this article, unless otherwise stated. 


\section{Background}

Radical prostatectomy (RP) or radiotherapy (RT) plus androgen-deprivation therapy (ADT) is recommended as standard treatment options for patients with high-risk localized prostate cancer (PCa) [1]. About 15-25\% localized PCa patients underwent RP would develop a biochemical recurrence (BCR); and tumor recurrence of high-risk $\mathrm{PCa}$ after RP has always been a concern of clinicians [2]. To achieve better prognosis, RT is always recommended for patients with adverse pathological features (APFs) after $\mathrm{RP}$, including extracapsular extension, seminal vesicle invasion, positive surgical margins (PSM), and high Gleason score (GS). Emerging evidence also indicates that postoperative RT could significantly control the local recurrence of tumor and reduce the risk of distant metastasis [3].

According to the timing and circumstances of the procedures, postoperative RT is divided into adjuvant radiotherapy (ART) and salvage radiotherapy (SRT). ART is given to patients with high risk of recurrence and an undetectable prostate-specific antigen (PSA) after prostatectomy due to APFs prior to recurrence. However, SRT is administrated to patients with an undetectable PSA that becomes subsequently detectable and increases on 2 measurements or a PSA that remains persistently detectable after RP $[4,5]$.

Even though several randomized controlled trials (RCTs) have previously indicated that patients with APFs received ART could achieve a better biochemical recurrence-free survival (BRFS) when compared to initial observation [6-8], the results from the National Cancer Data Base showed that the number of patients with APFs received post-prostatectomy ART was declining [9]. Concerns about the toxicity of radiotherapy, the tendency to choose salvage treatment after BCR and patient preference might explain this phenomenon [9]. Up to now, there is still no determined consensus on the pros and cons of these two therapies. As the optimal timing of postoperative RT remains controversial, we conducted this systematic review and meta-analysis to elevate the efficacy and the prognosis benefits of ART and SRT.

\section{Methods}

\section{Search strategy}

The search strategy was initiated by two reviewers respectively. To retrieve the required literature as completely as possible, a great number of databases have been searched, included PubMed (from 1950 to June 2019), EMBASE (using Ovid as the main search engine from 1974 to June 2019), Web of Science (from 1900 to June 2019) and the Cochrane Library electronic databases (from 1948 to June 2019). Combinations of the following MeSH and keywords were used in databases: (prostate neoplasms or prostate cancer or prostatic cancer) and (adjuvant radiotherapy or adjuvant RT) and (salvage radiotherapy or salvage RT).

\section{Inclusion and exclusion criteria}

The criteria for included studies were: (1) all potential studies concerning the comparison of the prognosis of postoperative ART and SRT; (2) articles published in English; (3) at least one of the following outcomes was reported: overall survival (OS), BRFS and distant metastases-free survival (DMFS).

All patients must undergo RP and had at least one of APFs, including PSM, extracapsular extension, seminal vesicle invasion, and high GS. The specific eligibility criteria for the ART group were as follows: (1) postoperative RT was initiated when serum PSA was undetectable; (2) RT should be performed within 6 months after RP. Correspondingly, the SRT group should meet the following conditions: (1) RT was started when serum PSA rising constantly from undetectable level; (2) patients have been shown to develop PSA recurrence.

Conference abstracts which did not provide enough information were excluded. Case reports, review articles and editorial comments were not in our consideration. Neoadjuvant therapy should not be administered to these patients. The patient had other malignancies other than prostate cancer should be also ruled out.

\section{Data extraction and quality assessment}

Two authors carried out the procedure of data extraction independently. The titles and abstracts of articles retrieved by the proposed strategy firstly were screened to rule out irrelevant articles. Then, the full texts of selected articles were elevated in complying with the inclusion and exclusion criteria. The necessary data of the finally included articles were extracted, included the type of study, authors, publication year, the characteristics of participants in the ART and SRT groups (number, age, GS, staging, and follow-up time), outcomes (OS, BRFS, DMFS and related hazard ratio (HR)), etc.

The Newcastle-Ottawa scale (NOS), included three items: Selection, Comparability, and Outcome, was used to elevate the methodological quality of each study [10]. Each article was scored on a scale of 0 to 9 . A study that achieved a score of 8 or 9 was considered high quality and a score of 5 to 7 were regarded as moderate quality [11]. Discussion and consultation assisted in resolving an existed disagreement between two authors during the procedure.

\section{Outcomes of interest}

The primary outcomes were OS and BRFS. The secondary outcome was DMFS. OS was defined as the time from RP/RT to death, irrespective of the reason of death. BRFS was calculated from RP/RT to a detectable PSA 
value, or a serum PSA $>0.2 \mathrm{ng} / \mathrm{ml}$ on two consecutive detections for post-RP patients, or a rise of PSA $>0.2 \mathrm{ng} /$ $\mathrm{ml}$ above nadir for post-RT patients. DMFS was identified as the time from RP/RT to evidence of local recurrence or distant metastasis from imaging examination.

\section{Data synthesis and analysis}

The Review Manager software (RevMan version 5.3, The Cochrane Collaboration 2014) was used to analyze the data. Two reviewers input the data and performed the analysis. The other reviewers verified it in order to minimize the chance of error and bias.

As OS, BRFS and DMFS were time-to-event outcomes and were most appropriately analyzed using HR [12], we used HR between two survival distributions as a summary statistic. For a study which reported HR and corresponding 95\% confidence interval (CI), we extracted it directly. We also calculated these HRs and the corresponding 95\% CIs of the included studies which provided sufficient data using the methods outlined by Tierney and colleagues [12]. In accordance with the contract, an overall HR of less than 1 favored the ART group. The survival beneficial effect of ART compared to SRT was considered statistically significant if the 95\% CI of HR did not overlap 1 [13]. The reported odds ratio (OR) with 95\% CI was also calculated in the analysis.

The heterogeneity among the studies was assessed using the Chi-squared test and the $\mathrm{I}^{2}$ statistic. A $p$ value of $<0.1$ and an $\mathrm{I}^{2}$ value $>50 \%$ were considered as statistical heterogeneity. If significant heterogeneity was indicated, a random-effect model was used; instead, a fixed-effect model was used. Funnel plots would be used to investigate publication bias if enough studies were available.

5-year and 10-year OS and BRFS rates of these included studies were further extracted and a subgroup analysis was conducted in terms of the starting point of follow-up time. We also separately compared the survival benefits of ART with early salvage radiotherapy (ESRT), which was defined as RT administered at a postoperative serum $\mathrm{PSA} \leq 0.5 \mathrm{ng} / \mathrm{ml}$.

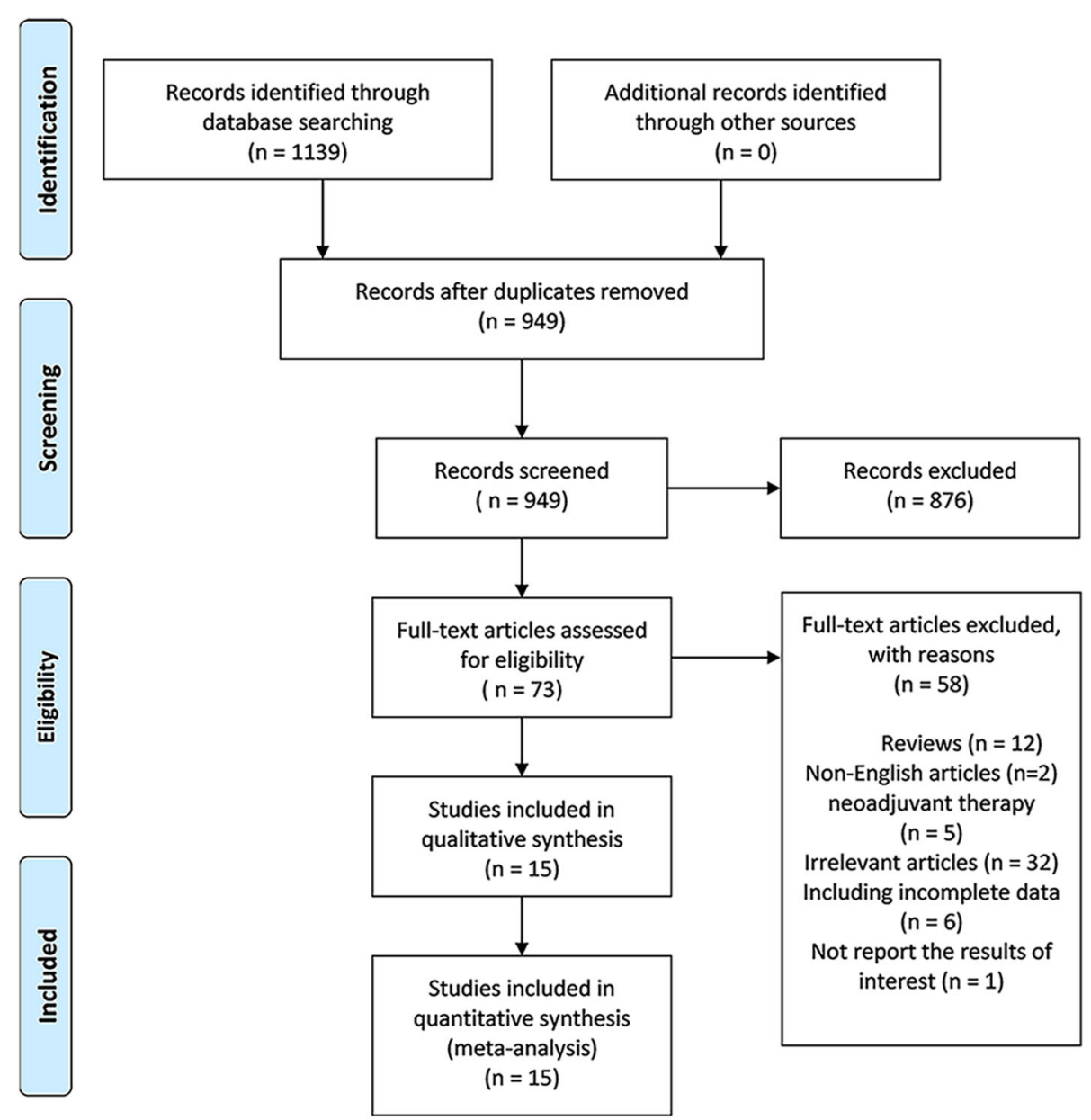

Fig. 1 Flow diagram of qualified studies 


\section{Results}

\section{Characteristics of included studies}

According to our search strategy, 1139 articles were screened. A total of sixteen studies met with the predefined inclusion criteria; however, fifteen studies were finally included in the meta-analysis [14-28]. One study was excluded due to lack of necessary data, failure to report the results of interest, and unsuccessful HR extract [29]. Five studies were excluded because some subjects received neoadjuvant therapy [30-34]. Since two clinical trials paid more attention to the comparison of ART and wait-and-see, but the percentage of patients who received SRT in the observation group was very small, they were both not in our consideration [7, 35]. The selection process of qualified studies was shown in Fig. 1.

All included articles were cohort studies published between 2002 and 2018. Seven studies took RP as the starting point of follow-up and reported corresponding results [14-18, 21, 22]. Six studies followed RT as the basis of follow-up and reported related results [19, 20, 23, 24, 27, 28]. The remaining two studies reported respectively results at different follow-up starting points [25, 26]. It was worth mentioning that four articles compared the prognosis of postoperative ART and ESRT [14-17]. The detailed description of these studies was shown in Table 1.

\section{Characteristics of patients}

A total of 5586 patients with APFs after RP were enrolled in this meta-analysis, including 2419 patients received ART and 3167 patients got SRT. Of those patients received SRT, 1253 patients specifically were stated that they were treated with ESRT. The age of subjects ranged from 59 to 66 years old in different studies. The PSM rate of patients in the ART group ranged from 50 to $96 \%$, while it ranged from 23 to $87 \%$ in the SRT group. Detailed information of included patients was summarized in Table 2 .

\section{Overall survival}

The HRs and corresponding 95\% CIs of OS in five articles were pooled and the results showed that OS in the ART group was better than that in the SRT group (HR: 0.58 ; 95\%CI: $0.42-0.79 ; p=0.0006)$ with no statistically significant heterogeneity $\left(\mathrm{I}^{2}=0, p=0.64\right)[15-17,21,23]$ (Fig. 2a). Subgroup analysis also indicated a statistically significant difference between postoperative patients received ART and SRT when follow-up time was calculated from RP (HR: 0.55; 95\%CI: 0.39-0.78; $p=0.0007$ ). No significant heterogeneity was found among studies $\left(\mathrm{I}^{2}=0, p=0.57\right)$. However, the subgroup analysis of follow-up from RT was not applicable (Fig. 2a).

We also compared the OS of patients who received postprostatectomy ART versus ESRT, which were extracted from three studies [15-17]. The meta-analysis of these studies showed that ART still had an advantage over ESRT in terms of OS (HR:0.59; 95\%CI: 0.40-0.87; $p=0.008$ ). Likewise, there was no evidence to reveal a significant heterogeneity between studies $\left(\mathrm{I}^{2}=0, p=0.60\right)$ (Fig. $2 \mathrm{~B}$ ).

Table 1 Characteristics of included studies

\begin{tabular}{|c|c|c|c|c|c|c|c|c|}
\hline \multirow[t]{2}{*}{ Reference } & \multirow[t]{2}{*}{ Study type } & \multirow[t]{2}{*}{ Country } & \multirow{2}{*}{$\begin{array}{l}\text { Study } \\
\text { period }\end{array}$} & \multicolumn{3}{|c|}{ Sample size (n) } & \multicolumn{2}{|l|}{ Follow-up time (months) } \\
\hline & & & & Total & ART & SRT & ART & SRT \\
\hline Briganti 2012 & match-controlled & Italy & $1991-2007$ & 780 & 390 & 390 & Median (IQR):71.9 (39-103) & Median (IQR):41 (10-60) \\
\hline Buscariollo 2017 & retrospective cohort & United States & 1992-2013 & 401 & 171 & 230 & Median (IQR):89 (55-158) & Median (IQR):96 (63-130) \\
\hline Fossati 2016 & retrospective cohort & Italy & 1996-2009 & 510 & 243 & 267 & Median (IQR):94 (53-126) & Median (IQR):92 (70-136) \\
\hline Hwang 2018 & retrospective cohort & United States & 1987-2013 & 732 & 366 & 366 & Median (IQR):65.8 (40-107) & Median (IQR):73.3 (44.9-106.6) \\
\hline Borghetti 2017 & retrospective cohort & Italy & 1999-2012 & 258 & 127 & 131 & Overall median:50.7 & - \\
\hline Hervas 2017 & retrospective cohort & Spain & $1991-2011$ & 702 & 223 & 479 & $\begin{array}{l}\text { Overall median (range):34 } \\
(3-141)\end{array}$ & - \\
\hline Mishra 2015 & retrospective cohort & United States & 1990-2009 & 186 & 74 & 112 & $\begin{array}{l}\text { Overall median (range):103 } \\
(30-247)\end{array}$ & - \\
\hline Hsu 2015 & retrospective cohort & United States & 1995-2009 & 305 & 76 & 229 & $\begin{array}{l}\text { Overall median (range):74 } \\
(7-256)\end{array}$ & - \\
\hline Tilki 2016 & retrospective cohort & Germany & $2005-2013$ & 718 & 213 & 505 & $\begin{array}{l}\text { Overall median (IQR):33.8 } \\
(17.1-49.0)\end{array}$ & - \\
\hline Detti 2012 & retrospective cohort & Italy & 1995-2010 & 307 & 203 & 104 & Mean \pm SD:3.3 \pm 2.3 & Mean \pm SD:4.5 \pm 2.5 \\
\hline Ost 2011 & match-controlled & Belgium & 1999-2009 & 178 & 89 & 89 & Median (range):36 (3-120) & Median (range):36 (3-120) \\
\hline Trabulsi 2008 & matched-Control & United States & 1987-2002 & 192 & 96 & 96 & Median (range):97 (30-207) & Median (range):94 (26-190) \\
\hline Tsien 2003 & retrospective cohort & United States & 1986-1997 & 95 & 38 & 57 & Median (range):10.1 (4.8-14.5) & Median (range):8.8 (2.0-17.0) \\
\hline Taylor 2003 & retrospective cohort & United States & 1988-1998 & 146 & 75 & 71 & Median:68 & Median:39 \\
\hline
\end{tabular}


Table 2 Characteristic of all included patients

\begin{tabular}{|c|c|c|c|c|c|c|c|c|}
\hline Reference & Group & Age & $\begin{array}{l}\text { Gleason score } n \\
(\leq 6 / 7 / \geq 8)\end{array}$ & $\begin{array}{l}\text { Pathologic } \\
\text { T stage (n) }\end{array}$ & $\begin{array}{l}\text { Preoperative PSA (ng/ } \\
\mathrm{ml} \text { ) }\end{array}$ & $\begin{array}{l}\text { Pre-RT PSA } \\
(\mathrm{ng} / \mathrm{ml})\end{array}$ & $\begin{array}{l}\text { PSM } \\
\text { n (\%) }\end{array}$ & $\begin{array}{l}\text { Radiation dose } \\
\text { (Gy) }\end{array}$ \\
\hline \multirow[t]{2}{*}{$\begin{array}{l}\text { Briganti } \\
2012\end{array}$} & ART & $\begin{array}{l}\text { Median (IQR): } \\
64(60-68)\end{array}$ & $160 / 185 / 45$ & $\begin{array}{l}\text { T3a:261 T3b: } \\
129\end{array}$ & $\begin{array}{l}\text { Median(IQR): } \\
10(6.7-16.1)\end{array}$ & $\begin{array}{l}\text { Median(IQR): } \\
0(0-0)\end{array}$ & $\begin{array}{l}245 \\
(62.8)\end{array}$ & $\begin{array}{l}\text { Median(IQR): } \\
65 \text { (60-70) }\end{array}$ \\
\hline & ESRT & $\begin{array}{l}\text { Median (IQR): } \\
65(61-69)\end{array}$ & $163 / 173 / 54$ & $\begin{array}{l}\text { T3a:274 T3b: } \\
116\end{array}$ & $\begin{array}{l}\text { Median(IQR): } \\
10(6.3-14.7)\end{array}$ & $\begin{array}{l}\text { Median(IQR): } \\
0.2(0.1-0.3)\end{array}$ & $\begin{array}{l}238 \\
(61.0)\end{array}$ & $\begin{array}{l}\text { Median(IQR): } \\
66(66-66)\end{array}$ \\
\hline \multirow[t]{2}{*}{$\begin{array}{l}\text { Buscariollo } \\
2017\end{array}$} & ART & $\begin{array}{l}\text { Median (IQR): } \\
60(54-65)\end{array}$ & $31 / 83 / 56$ & $\begin{array}{l}\text { T2:43 } \\
\text { T3:128 }\end{array}$ & $\begin{array}{l}\text { Median(IQR): } \\
6(5-10)\end{array}$ & $<0.1$ & $\begin{array}{l}143 \\
(83.6)\end{array}$ & $\begin{array}{l}\text { Median(IQR): } \\
61.2(61.2,64.8)\end{array}$ \\
\hline & ESRT & $\begin{array}{l}\text { Median (IQR): } \\
59(54-63)\end{array}$ & $43 / 142 / 45$ & $\begin{array}{l}\text { T2:90 } \\
\text { T3:140 }\end{array}$ & $\begin{array}{l}\text { Median(IQR): } \\
6(5-10)\end{array}$ & $\begin{array}{l}\text { Median(IQR): } \\
0.3(0.2-0.4)\end{array}$ & $\begin{array}{l}163 \\
(70.9)\end{array}$ & $\begin{array}{l}\text { Median(IQR): } \\
64.8(64.8,64.8)\end{array}$ \\
\hline \multirow[t]{2}{*}{$\begin{array}{l}\text { Fossati } \\
2016\end{array}$} & ART & $\begin{array}{l}\text { Median (IQR): } \\
64 \text { (61-69) }\end{array}$ & $57 / 120 / 66$ & $\begin{array}{l}\text { T3a:137 } \geq \\
\text { T3b:106 }\end{array}$ & $\begin{array}{l}\text { Median(IQR): } \\
9.3(6.2-15.8)\end{array}$ & $\begin{array}{l}\text { Median(IQR): } \\
0(0-0)\end{array}$ & $\begin{array}{l}181 \\
(74)\end{array}$ & $\begin{array}{l}\text { Median(IQR): } \\
60(60-65)\end{array}$ \\
\hline & ESRT & $\begin{array}{l}\text { Median (IQR): } \\
65(60-70)\end{array}$ & 49/147/71 & $\begin{array}{l}\text { T3a:168 } \geq \\
\text { T3b:99 }\end{array}$ & $\begin{array}{l}\text { Median(IQR): } \\
9.8(6.3-14.8)\end{array}$ & $\begin{array}{l}\text { Median(IQR): } \\
0.2(0.1-0.3)\end{array}$ & $\begin{array}{l}138 \\
(52)\end{array}$ & $\begin{array}{l}\text { Median(IQR): } \\
67(66-67)\end{array}$ \\
\hline \multirow[t]{2}{*}{$\begin{array}{l}\text { Hwang } \\
2018\end{array}$} & ART & $\begin{array}{l}\text { Median (IQR): } \\
60 \text { (55-65) }\end{array}$ & $50 / 210 / 106$ & $\begin{array}{l}\text { T2:98 } \\
\text { T3:268 }\end{array}$ & UC & $<0.1$ & $\begin{array}{l}313 \\
(85.8)\end{array}$ & $\begin{array}{l}\text { Median(IQR): } \\
64.8(61.2-66.0)\end{array}$ \\
\hline & ESRT & $\begin{array}{l}\text { Median (IQR): } \\
61 \text { (54.6-65.3) }\end{array}$ & $33 / 209 / 124$ & $\begin{array}{l}\text { T2:109T3: } \\
257\end{array}$ & UC & $\begin{array}{l}\text { Median(IQR): } \\
0.3(0.2-0.4)\end{array}$ & $\begin{array}{l}318 \\
(86.9)\end{array}$ & $\begin{array}{l}\text { Median(IQR): } \\
66.0(64.8-70.0)\end{array}$ \\
\hline \multirow[t]{2}{*}{$\begin{array}{l}\text { Borghetti } \\
2017\end{array}$} & ART & $\begin{array}{l}\text { Overall median } \\
\text { (range): } 65 \text { (42-78) }\end{array}$ & $24 / 60 / 43$ & $\begin{array}{l}\mathrm{T} 2: 20 \geq \mathrm{T} 3: \\
107\end{array}$ & UC & UC & $\begin{array}{l}99 \\
(78.0)\end{array}$ & $U C$ \\
\hline & SRT & & $42 / 57 / 32$ & $\begin{array}{l}\text { T2:50 } \\
\geq T 3: 81\end{array}$ & UC & UC & $\begin{array}{l}80 \\
(61.1)\end{array}$ & UC \\
\hline \multirow[t]{2}{*}{$\begin{array}{l}\text { Hervas } \\
2017\end{array}$} & ART & $\begin{array}{l}\text { Mean (range): } \\
62.7(43.0-75.0)\end{array}$ & $199(\leq 7) / 20$ & $\begin{array}{l}\leq \mathrm{T} 2: 83 \geq \mathrm{T} 3: \\
124\end{array}$ & UC & $\leq 0.5$ & $\begin{array}{l}156 \\
(70.9)\end{array}$ & UC \\
\hline & SRT & $\begin{array}{l}\text { Mean (range): } \\
64.8 \text { (42.0-82.0) }\end{array}$ & $393(\leq 7) / 47$ & $\begin{array}{l}\leq \mathrm{T} 2: 272 \geq \\
\mathrm{T} 3: 172\end{array}$ & UC & UC & $\begin{array}{l}214 \\
(47.5)\end{array}$ & UC \\
\hline \multirow[t]{2}{*}{$\begin{array}{l}\text { Mishra } \\
2015\end{array}$} & ART & Median:59 & $12 / 40 / 19$ & UC & UC & Median: $<0.1$ & $\begin{array}{l}60 \\
(81.1)\end{array}$ & Median:66 \\
\hline & SRT & Median:63 & $22 / 49 / 33$ & UC & UC & Median:0.6 & $\begin{array}{l}86 \\
(76.8)\end{array}$ & Median:66.6 \\
\hline \multirow[t]{2}{*}{ Hsu 2015} & ART & UC & $14 / 34 / 26$ & $\begin{array}{l}\leq \mathrm{T} 2: 12 \geq \mathrm{T} 3: \\
64\end{array}$ & UC & $<0.1$ & $50(79)$ & UC \\
\hline & SRT & UC & $22 / 118 / 83$ & $\begin{array}{l}\leq \mathrm{T} 2: 86 \geq \mathrm{T} 3: \\
143\end{array}$ & UC & $\begin{array}{l}\text { Median(IQR): } \\
0.5(0.3-1.0)\end{array}$ & $\begin{array}{l}149 \\
(86)\end{array}$ & UC \\
\hline \multirow[t]{2}{*}{ Tilki 2016} & ART & $\begin{array}{l}\text { Median (IQR): } \\
65(60-70)\end{array}$ & 0/116/97 & T2:9 T3:204 & $\begin{array}{l}\text { Median(IQR): } \\
12(7.8-25.7)\end{array}$ & UC & $\begin{array}{l}171 \\
(80.3)\end{array}$ & range:60-70 \\
\hline & SRT & $\begin{array}{l}\text { Median (IQR): } \\
66(61-70)\end{array}$ & $1 / 340 / 163$ & T2:63 T3:441 & $\begin{array}{l}\text { Median(IQR): } \\
11(7.0-18.9)\end{array}$ & UC & $\begin{array}{l}212 \\
(42)\end{array}$ & range:60-70 \\
\hline \multirow[t]{2}{*}{ Detti 2012} & ART & $\begin{array}{l}\text { Mean } \pm \text { SD: } \\
65.1 \pm 7.3\end{array}$ & $44 / 77 / 82$ & $\begin{array}{l}\mathrm{T} 2: 22 \\
\geq \mathrm{T} 3: 181\end{array}$ & $\begin{array}{l}\text { Mean } \pm \text { SD: } \\
0.10 \pm 0.28\end{array}$ & $\begin{array}{l}\text { Mean } \pm \text { SD: } \\
0.47 \pm 1.73\end{array}$ & $\begin{array}{l}101 \\
(49.8)\end{array}$ & $\begin{array}{l}\text { Mean } \pm \text { SD: } \\
66.2 \pm 4.1\end{array}$ \\
\hline & SRT & $\begin{array}{l}\text { Mean } \pm \text { SD: } \\
67.0 \pm 6.0\end{array}$ & $25 / 26 / 53$ & $\begin{array}{l}\mathrm{T} 2: 23 \geq \mathrm{T} 3: \\
81\end{array}$ & $\begin{array}{l}\text { Mean } \pm \text { SD: } \\
0.85 \pm 0.52\end{array}$ & $\begin{array}{l}\text { Mean } \pm \text { SD: } \\
1.73 \pm 3.19\end{array}$ & $\begin{array}{l}24 \\
(23.1)\end{array}$ & $\begin{array}{l}\text { Mean } \pm \text { SD: } \\
66.8 \pm 4.1\end{array}$ \\
\hline \multirow[t]{2}{*}{ Ost 2011} & ART & $\begin{array}{l}\text { Median(range): } \\
63(51-77)\end{array}$ & $\begin{array}{l}\text { 64(including } 3+4) / \\
25 \text { (including } 4+3 \text { ) }\end{array}$ & $\begin{array}{l}T 2: 21 \\
\geq T 3: 68\end{array}$ & $\begin{array}{l}\text { Median(range): } 10.0 \\
\text { (3.0-47.9) }\end{array}$ & $<0.2$ & $68(76)$ & Median:74 \\
\hline & SRT & $\begin{array}{l}\text { Median(range): } \\
64(42-75)\end{array}$ & $\begin{array}{l}64 \text { (including } 3+4 \text { )/ } \\
25 \text { (including } 4+3 \text { ) }\end{array}$ & $\begin{array}{l}\mathrm{T} 2: 21 \\
\geq \mathrm{T} 3: 68\end{array}$ & $\begin{array}{l}\text { Median(range): } 10.0 \\
(3.5-148)\end{array}$ & $\geq 0.2$ & $59(66)$ & Median:76 \\
\hline \multirow[t]{2}{*}{$\begin{array}{l}\text { Trabulsi } \\
2008\end{array}$} & ART & $\begin{array}{l}\text { Median(range): } \\
62.0(42-76)\end{array}$ & $22 / 17 / 57$ & $\geq \mathrm{T} 3: 96$ & $\begin{array}{l}\text { Median(range): } \\
8.3(1.1-65.9)\end{array}$ & $<0.2$ & $80(83)$ & $\begin{array}{l}\text { Median(range): } \\
60(50-70)\end{array}$ \\
\hline & SRT & $\begin{array}{l}\text { Median(range): } \\
63.0(47-75)\end{array}$ & $22 / 17 / 57$ & $\geq \mathrm{T} 3: 96$ & $\begin{array}{l}\text { Median(range): } \\
9.0(1.7-39)\end{array}$ & $\begin{array}{l}\text { Median(range): } \\
0.7(0.2-2)\end{array}$ & $80(83)$ & $\begin{array}{l}\text { Median(range): } \\
64.8(59-70)\end{array}$ \\
\hline \multirow[t]{2}{*}{ Tsien 2003} & ART & $\begin{array}{l}\text { Median(range): } \\
63.0(43.8-75.7)\end{array}$ & $11 / 16 / 8$ & $\geq \mathrm{T} 3: 36$ & $\begin{array}{l}\text { Median(range): } 11.6 \\
(1.1-99.6)\end{array}$ & UC & $34(89)$ & $\begin{array}{l}\text { Median(range): } \\
64.0 \text { (59.4-69.0) }\end{array}$ \\
\hline & SRT & $\begin{array}{l}\text { Median(range): } \\
64.2(42.1-78.6)\end{array}$ & $17 / 27 / 8$ & $\geq \mathrm{T} 3: 38$ & $\begin{array}{l}\text { Median(range): } 13.3 \\
(0.2-120.0)\end{array}$ & $\begin{array}{l}\text { Median(range): } \\
1.2(0.2-18.4)\end{array}$ & $27(47)$ & $\begin{array}{l}\text { Median(range): } \\
65.0(60.0-75.0)\end{array}$ \\
\hline Taylor 2003 & ART & median:60 & $9 / 35 / 30$ & $\leq T 2: 27 \geq T 3:$ & Median:11 & Median:<0.1 & $73(96)$ & Median(range): \\
\hline
\end{tabular}


Table $\mathbf{2}$ Characteristic of all included patients (Continued)

\begin{tabular}{|c|c|c|c|c|c|c|c|c|}
\hline Reference & Group & Age & $\begin{array}{l}\text { Gleason score } n \\
(\leq 6 / 7 / \geq 8)\end{array}$ & $\begin{array}{l}\text { Pathologic } \\
\text { T stage (n) }\end{array}$ & $\begin{array}{l}\text { Preoperative PSA (ng/ } \\
\mathrm{ml} \text { ) }\end{array}$ & $\begin{array}{l}\text { Pre-RT PSA } \\
(\mathrm{ng} / \mathrm{ml})\end{array}$ & $\begin{array}{l}\text { PSM } \\
\mathrm{n}(\%)\end{array}$ & $\begin{array}{l}\text { Radiation dose } \\
\text { (Gy) }\end{array}$ \\
\hline & & & & 48 & & & & $60(51-70)$ \\
\hline & SRT & UC & $18 / 27 / 24$ & $\begin{array}{l}\leq \mathrm{T} 2: 12 \geq \mathrm{T} 3 \\
59\end{array}$ & UC & UC & UC & $\begin{array}{l}\text { Median(range): } \\
70(60-78)\end{array}$ \\
\hline \multirow[t]{2}{*}{$\begin{array}{l}\text { Kalapurakal } \\
2002\end{array}$} & ART & $\begin{array}{l}\text { Overall median } \\
\text { (range): } 60 \text { (48-78) }\end{array}$ & Overall $66(\leq 7) / 10$ & UC & $\begin{array}{l}\text { Overall median(range): } \\
12.0 \text { (4-82) }\end{array}$ & UC & $\begin{array}{l}\text { overall } \\
40(53)\end{array}$ & $\begin{array}{l}\text { Median(range): } \\
60(60-65)\end{array}$ \\
\hline & SRT & & & UC & & $\begin{array}{l}\text { Median(range): } \\
0.5(0.2-6.5)\end{array}$ & & $\begin{array}{l}\text { Median(range): } \\
65(60-70)\end{array}$ \\
\hline
\end{tabular}

UC: unclear

Three studies reported 5-year OS rates of the ART and SRT groups [18-20], and four studies reported 10year OS rates of these patients $[15,18,20,21]$. The pooled results of 5-year OS rate showed there was a significant statistical difference between the ART group and the SRT group (OR: 0.19; 95\%CI: $0.07-0.49 ; p=$ $0.0006 ; \mathrm{I}^{2}=45 \%$ ) (Fig. 3a). Similarly, subgroup analysis presented a statistically significant difference between the ART group and the SRT group when follow-up from RT (OR: 0.08; 95\%CI: 0.02-0.44; $p=0.003 ; \mathrm{I}^{2}=20 \%$ ) (Fig. 3a). But the subgroup analysis of follow-up from RP was not applicable (Fig. 3a).

Nevertheless, the pooled result of 10 -year OS rate suggested there was no significant statistical difference between the ART group and the SRT group (OR:0.70; 95\%CI: $0.48-1.03 ; p=0.07 ; \mathrm{I}^{2}=30 \%$ ) (Fig. 3B). Subgroup analysis indicated that there was also no statistically significant difference between two groups in terms of 10-year OS rate for studies calculated from RP (OR: 0.81; 95\%CI: $0.53-1.23 ; p=0.33 ; \mathrm{I}^{2}=0 \%$ ) (Fig. 3b). The subgroup analysis of follow-up from RT was also not applicable (Fig. 3b).

\section{Biochemical recurrence-free survival}

The HRs and corresponding 95\% CIs of BRFS between the ART and SRT groups were extracted from nine articles $[14,15,17,20,22-25,27]$. A meta-analysis of these studies by a random effect model showed that postoperative patients who received ART had better control of BCR compared to those received SRT (HR: 0.50; 95\%CI: $0.37-0.68 ; p<0.0001)$ with a statistically significant heterogeneity $\left(\mathrm{I}^{2}=76 \%, p<0.0001\right)$ (Fig. $\left.4 \mathrm{a}\right)$. The pooled result of data reported in four studies [14, 15, 17, 22], which took RP as the starting point of follow-up, showed

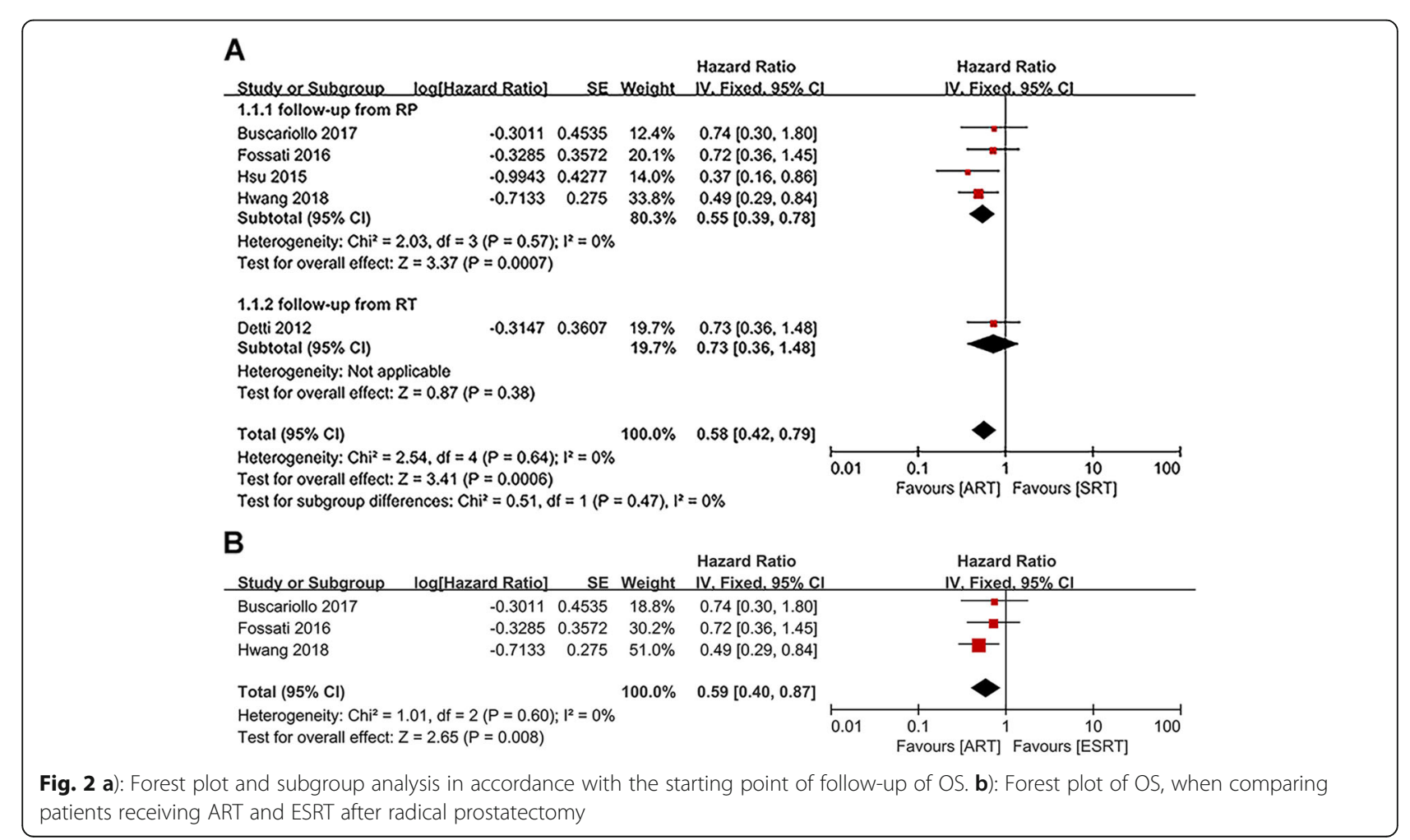




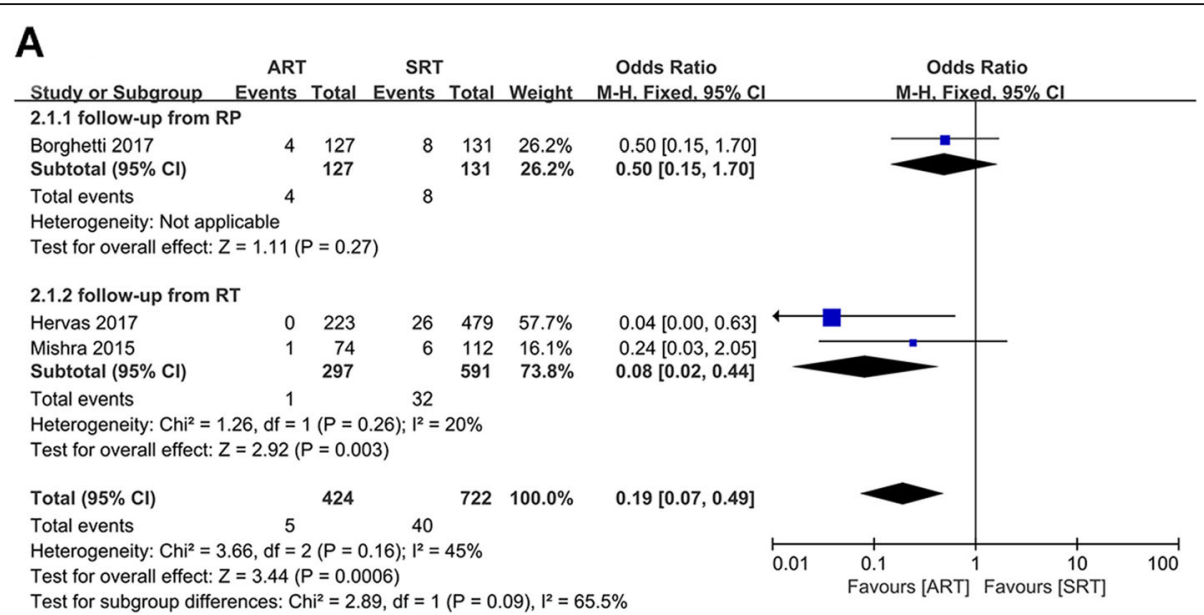

B

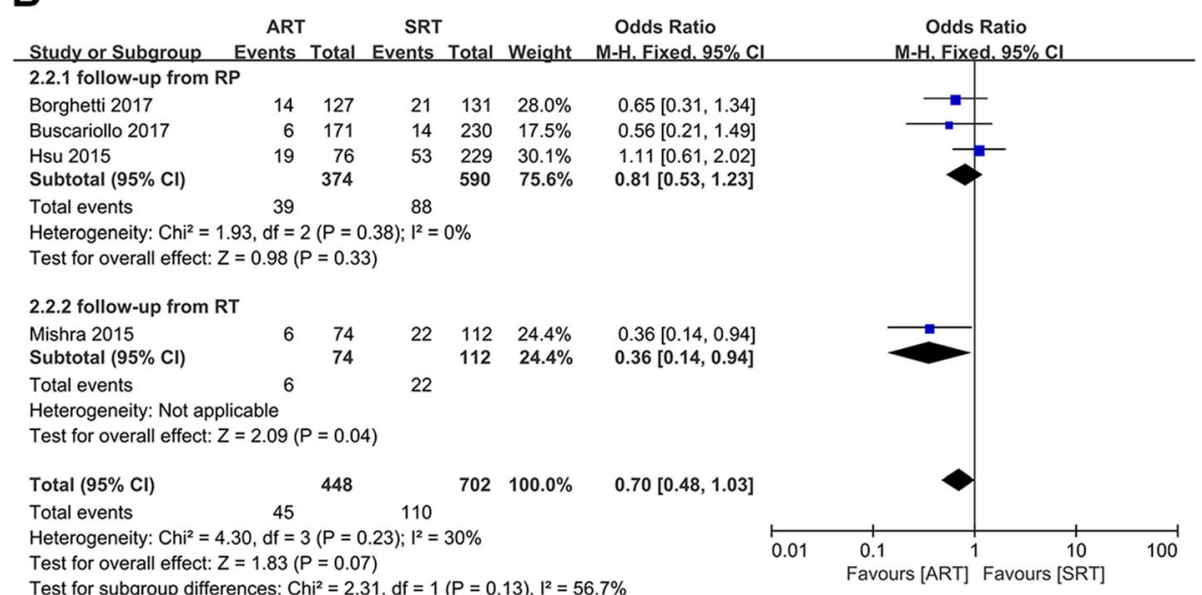

Fig. 3 a): Forest plot and subgroup analysis in accordance with the starting point of follow-up of 5-year OS rate. b): Forest plot and subgroup analysis in accordance with the starting point of follow-up of 10-year OS rate

there was a significant statistical difference between the ART and the SRT groups (HR: 0.56; 95\%CI: 0.34-0.92; $p=0.02$ ) with a statistically significant heterogeneity $\left(\mathrm{I}^{2}=88 \%, \mathrm{p}<0.0001\right)$ (Fig. 4a). The subgroup analysis of follow-up from RT revealed the similar tendency that the ART group owned a longer BCR process (HR: 0.45; 95\%CI: 0.31-0.66; $\mathrm{p}<0.0001$ ) (Fig. 4a). No statistically significant heterogeneity was found among these studies $\left(\mathrm{I}^{2}=46 \%, p=0.11\right)$ (Fig. 4a).

In the same way, we compared the BRFS of patients who received post-prostatectomy ART versus ESRT, which were obtained from three studies [14, 15, 17]. Conversely, the meta-analysis of these studies illustrated there was no statistical difference in BRFS between the ART and ESRT groups (HR: 0.60; 95\%CI: $0.31-1.17 ; p=0.14 ; \mathrm{I}^{2}=91 \%$ ). (Fig. 4b).

The 5-year BRFS rate of ART and SRT groups were reported in nine studies [14, 18-20, 24-28]. Data were pooled with a random-effect model since there was a significant difference in heterogeneity among these studies $\left(\mathrm{I}^{2}=78 \%, p<0.00001\right)$. The pooled result of the 5 -year BRFS rate showed there was a significant statistical difference between the ART and the SRT groups (OR: $0.46 ; 95 \% \mathrm{CI}: 0.30-0.71 ; p=0.0003$ ) (Fig. 5a). Of these studies, two calculated BRFS from the time of RP, five calculated BRFS from the time of RT and the remaining two reported 5-year BRFS rate both at these two different time points. The subgroup analysis showed 5 -year BRFS rate between the ART and SRT groups were comparable (OR: 0.90 ; 95\% CI: $0.60-1.35 ; p=0.60$ ) with no statistically significant heterogeneity $\left(\mathrm{I}^{2}=50 \%\right.$, $p=0.11$ ) when follow-up started from RP (Fig. 5a). However, the pooled data followed up from RT indicated that there was a significant statistical difference between the ART and the SRT groups (OR: 0.31; 95\%CI: 0.24-0.40; $p<0.00001$ ), which was consistent with the overall outcome (Fig. 5a). No significant heterogeneity was found among studies $\left(\mathrm{I}^{2}=0, p=0.78\right)$ (Fig. $\left.5 \mathrm{a}\right)$.

Three studies reported 10-year BRFS rate of these patients $[15,18,20]$. Random effect model meta-analysis was 


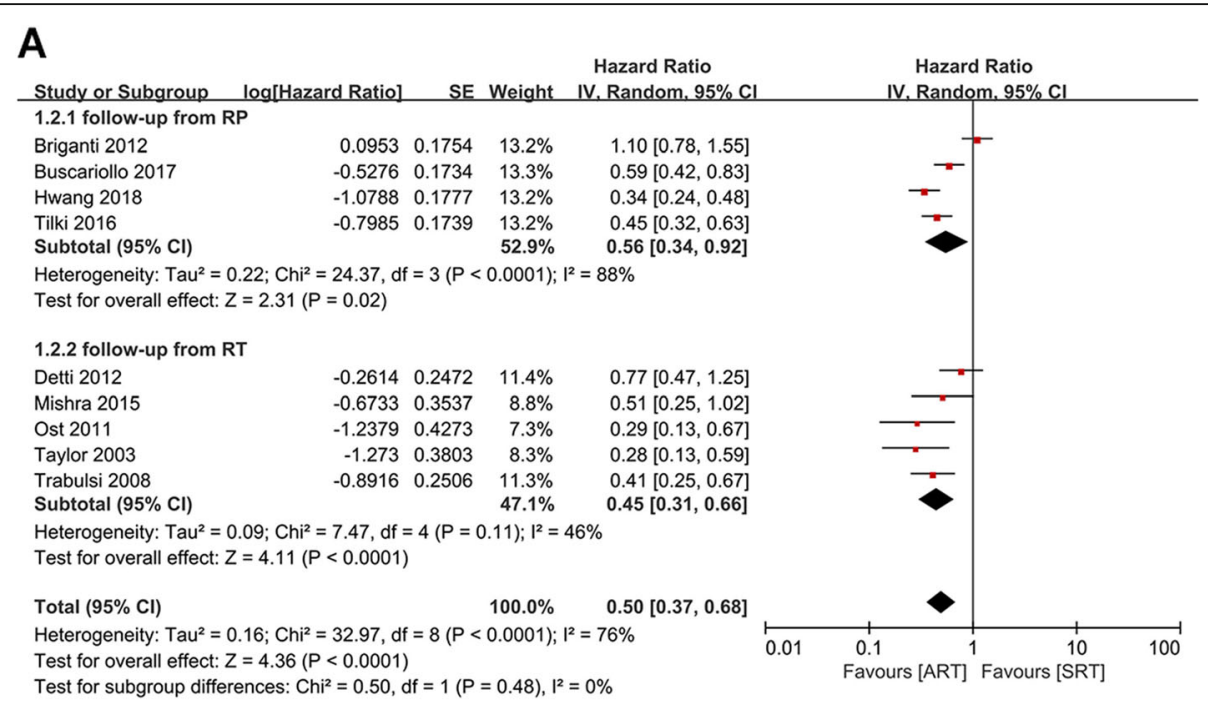

B

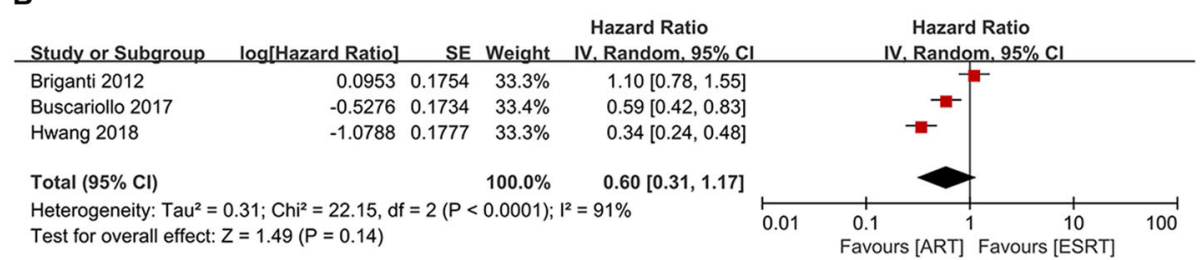

Fig. 4 a): Forest plot and subgroup analysis in accordance with the starting point of follow-up of BRFS. b): Forest plot of BRFS, when comparing patients receiving ART and ESRT after radical prostatectomy

conducted since there was a significant difference in heterogeneity among these studies $\left(\mathrm{I}^{2}=53 \% ; p=0.12\right)$. The pooled analysis showed that there was a significant statistical difference between the ART and the SRT groups (OR: 0.45; 95\%CI: 0.29-0.69; $p=0.0003$ ) (Fig. 5b). The subgroup analysis of follow-up started from RP indicated that there was also a significant statistical difference in 10-year BRFS rate between the ART and the SRT groups (OR: 0.55; 95\% CI: $0.40-0.76 ; \mathrm{p}=0.0003)$ with no statistically significant heterogeneity $\left(\mathrm{I}^{2}=0, p=0.88\right)$ (Fig. $\left.5 \mathrm{~b}\right)$.

\section{Distant metastases-free survival}

The HRs and corresponding 95\% CIs of DMFS were available in five studies [15-17, 20, 22]. A meta-analysis of these studies by a fixed effect model demonstrated that patients in the ART group had a lower risk of distant metastasis than those in the SRT group (HR: 0.51; 95\%CI: $0.36-0.71 ; p<0.0001)$ with no statistically significant heterogeneity $\left(\mathrm{I}^{2}=11 \%, p=0.34\right)$ (Fig. 6a). The subgroup analysis also produced a similar result that there was significant statistical difference in DMFS between the ART and the SRT groups (HR: 0.52; 95\%CI: 0.37$0.73 ; p=0.0002)$ without statistically significant heterogeneity $\left(\mathrm{I}^{2}=10 \%, \mathrm{p}=0.34\right)$ when follow-up from $\mathrm{RP}$ in four studies [15-17, 22] (Fig. 6a).
When compared the data from three studies focused on DMFS of patients who treated with postprostatectomy ART versus ESRT [15-17], the pooled analysis showed that there was a significant statistical difference between the ART and the ESRT groups (HR: $0.63 ; 95 \%$ CI: $0.42-0.95 ; p=0.03$ ). No significant heterogeneity was found among studies $\left(\mathrm{I}^{2}=0, p=0.6\right)$ (Fig. 6b).

\section{Quality assessment and publication bias}

The NOS scores of included studies ranged from 5 to 8 (median score: 7), which meant that all references rated at least moderate quality. The distribution of NOS scores of all included studies in this meta-analysis was presented in Table 3. A funnel plot of BRFS was made to evaluate the publication bias. No significant asymmetry can be found and it suggested there was no significant publication bias in this study (Fig. 7).

\section{Discussion}

As a matter of fact, $17-64 \%$ of patients who undergo RP would appear BCR, and up to one-third of men with BCR would develop metastatic diseases and eventually die of PCa [36]. Under these circumstances, the important role of postoperative radiotherapy is self-evident. According to the consensus reached by the American Urological Association 

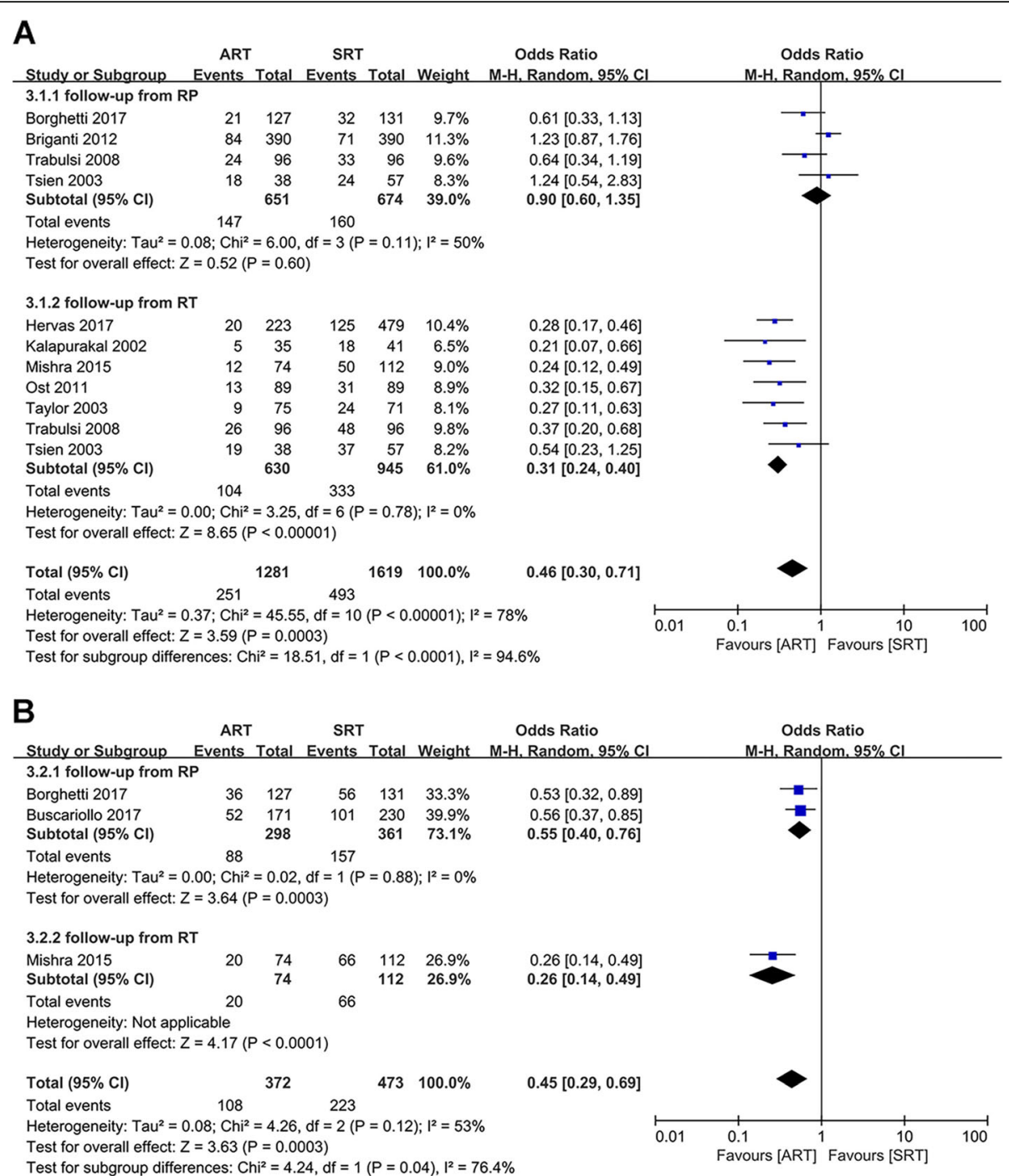

Fig. 5 a): Forest plot and subgroup analysis in accordance with the starting point of follow-up of 5-year BRFS rate. b): Forest plot and subgroup analysis in accordance with the starting point of follow-up of 10-year BRFS rate

(AUA) and the American Society of Radiation Oncology (ASTRO), patients with APFs should be informed that ART, compared to RP only, could reduce the risk of BCR, local recurrence, and clinical progression of cancer [4]. It also states that physicians should offer SRT to patients with $\mathrm{BCR}$ or local recurrence after RP, but without distant metastases [37]. So far, however, no definitive conclusion has been reached regarding the survival benefits of optimal timing of RT for patients with APFs following RP.

This systematic review and meta-analysis were designed to assist clinicians and patients to make optimal decisions by comparing the effect of ART and SRT on prognosis after RP. The pooled results of OS, BRFS, and DMFS revealed that ART could obtain better control of PCa disease and improve the survival outcomes when compared to SRT. ART also had advantages over SRT in both 5- and 10-year $\mathrm{BCR}$ rate. The analysis of 5-year OS rate demonstrated that ART still had survival advantages compared to SRT. However, ART and SRT were similar in 10-year OS rate. The loss of follow-up and censored data of these postoperative patients might account for these outcomes. Furthermore, with the development of $\mathrm{PCa}$, some patients who received SRT might be also treated with ADT, which would undoubtedly improve the efficacy of SRT. To sum up, it seems more advisable for patients with APFs after RP to receive ART to avoid missing the appropriate timing of radiotherapy.

Additionally, wait-and-see along with delayed RT until PSA starts to rise for postoperative patients with negative PSA could spare partial individuals from receiving unnecessary treatment since they might not develop a clinical recurrence. However, Oort et al. 

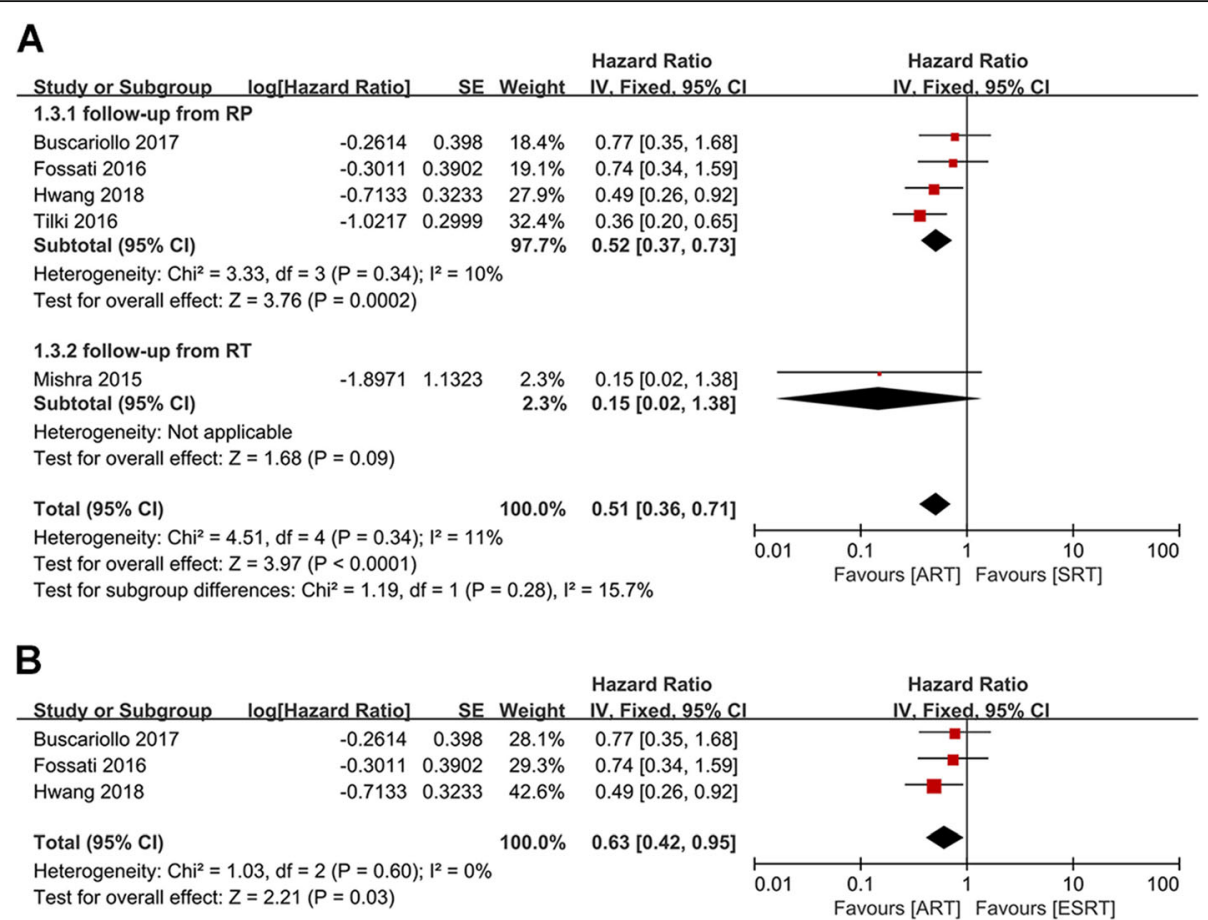

Fig. 6 a): Forest plot and subgroup analysis in accordance with the starting point of follow-up of DMFS. b): Forest plot of DMFS, when comparing patients receiving ART and ESRT after radical prostatectomy

reported that GS, pathologic stage, and PSM of RP specimens were the most powerful predictors of disease progression [38]. Swanson et al. also showed that positive seminal vesicles, Gleason sum score 8-10, extracapsular extension, and PSM were highly strong predictor of failure after prostatectomy [39]. Hence, there is no deny that the above prognostic factors must be considered comprehensively for clinicians and radiologists when planning ART for postoperative patients.

In fact, there is an ongoing RCT to compare outcomes of ART and ESRT, namely the radiotherapy assisted treatment and early rescue (RAVES) trial, which is led by the Trans Tasman Radiation Oncology

Table 3 Quality assessment of included studies

\begin{tabular}{|c|c|c|c|c|c|}
\hline Study & Selection & Comparability & Outcome & Total & Quality level \\
\hline Briganti 2012 & 3 & 2 & 3 & 8 & High \\
\hline Buscariollo 2017 & 3 & 2 & 3 & 8 & High \\
\hline Fossati 2016 & 3 & 1 & 3 & 7 & Moderate \\
\hline Hwang 2018 & 3 & 2 & 2 & 7 & Moderate \\
\hline Borghetti 2017 & 3 & 1 & 2 & 6 & Moderate \\
\hline Hervas 2017 & 3 & 0 & 3 & 6 & Moderate \\
\hline Mishra 2015 & 3 & 2 & 2 & 7 & Moderate \\
\hline Hsu 2015 & 3 & 2 & 3 & 8 & High \\
\hline Tilki 2016 & 3 & 2 & 3 & 8 & High \\
\hline Detti 2012 & 3 & 1 & 3 & 7 & Moderate \\
\hline Ost 2011 & 3 & 2 & 2 & 7 & Moderate \\
\hline Trabulsi 2008 & 3 & 2 & 2 & 7 & Moderate \\
\hline Tsien 2003 & 3 & 1 & 2 & 6 & Moderate \\
\hline Taylor 2003 & 3 & 1 & 2 & 6 & Moderate \\
\hline Kalapurakal 2002 & 3 & 1 & 1 & 5 & Moderate \\
\hline
\end{tabular}




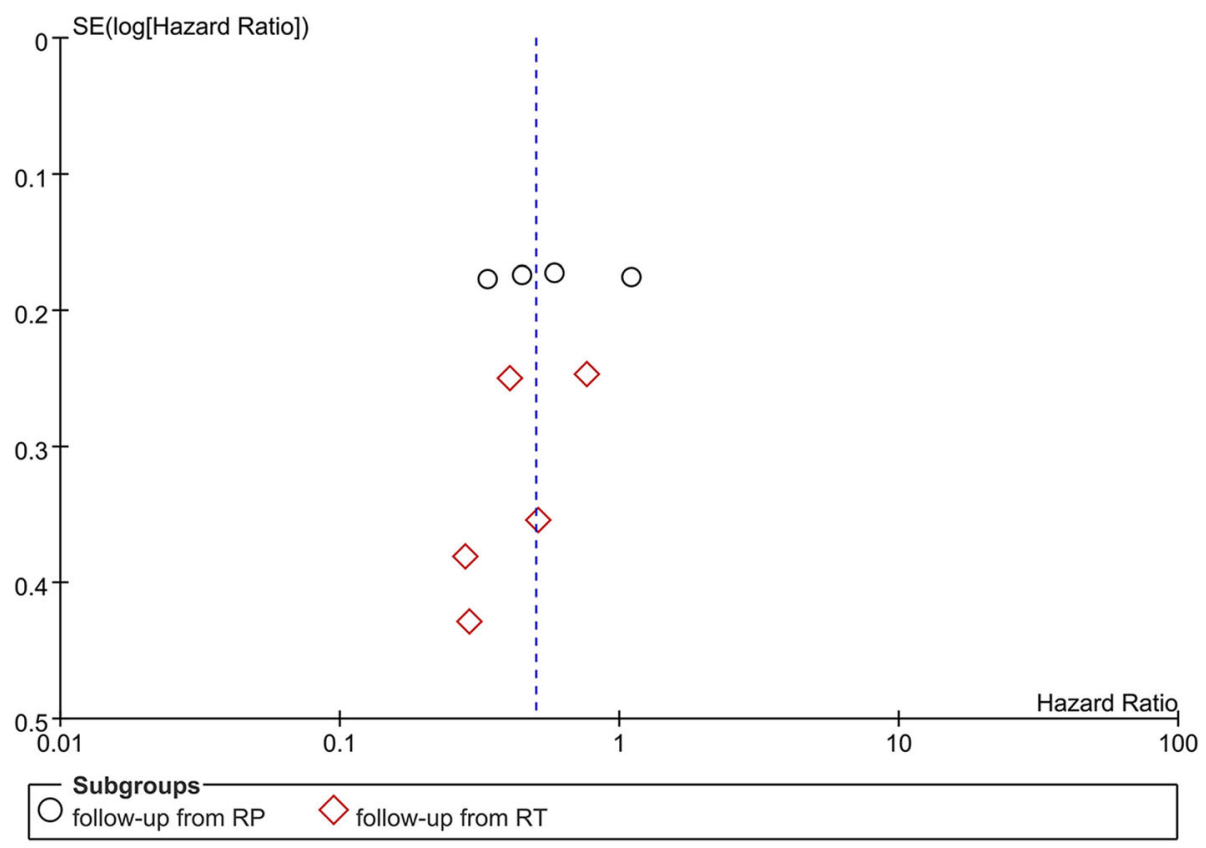

Fig. 7 Funnel plot constructed by biochemical recurrence-free survival (BRFS) of the included studies compared ART with SRT

Group (TROG), in collaboration with the Urological Society of Australia and New Zealand (USANZ), and the Australian and New Zealand Urogenital and Prostate Cancer Trials Group (ANZUP) [40]. Because of the specificity of prostate cancer progression, the results of this clinical trial might take a long time to be known. In order to get more comprehensive results, we also specifically analyzed the retrospective information about the outcomes of ART and ESRT in this meta-analysis. It suggested that ART was superior to ESRT on OS and DMFS, and similar to ESRT on BRFS.

In our review, the limitations that we should discuss are as follows. First, the information was obtained from a number of retrospective studies, which might introduce confounding data and produce bias. Second, all studies were carried out between 1987 and 2013 in different countries. The development of RT and the different implementation standards of RT in different regions could affect the prognosis of patients after RP. Main types of RT used to treat PCa including external beam radiation therapy and brachytherapy. Even patients from the same region would receive RT in different ways, which might affect the final results. In addition, most of the included studies paid more attention to biochemical control or OS of postoperative patients treated with ART and SRT and rarely reported adverse effects caused by these two radiotherapies. Finally, some subjects in the included studies received RT along with ADT, which might affect the differences between the two types of radiation.

\section{Conclusion}

According to this meta-analysis, ART was superior to SRT (including ESRT) on OS and DMFS and could be served as a preferential treatment for patients with APFs after RP to achieve a better prognosis. Certainly, highquality, multicenter RCTs are expecting to confirm the outcomes of our meta-analysis in the future.

\section{Abbreviations \\ ADT: Androgen-deprivation therapy; APFs: Adverse pathological features; ART: Adjuvant radiotherapy; BCR: Biochemical recurrence; BRFS: Biochemical recurrence-free survival; Cl: Confidence interval; DMFS: Distant metastases- free survival; ESRT: Early salvage radiotherapy; GS: Gleason score; HR: Hazard ratio; NOS: Newcastle-Ottawa scale; OR: Odds ratio; OS: Overall survival; PCa: Prostate cancer; PSA: Prostate-specific antigen; PSM: Positive surgical margins; RCTs: Randomized controlled trials; RP: Radical prostatectomy; RT: Radiotherapy; SRT: Salvage radiotherapy}

\section{Acknowledgments \\ Not applicable.}

\section{Author contribution}

$R T, J D$, and PS designed the study. RT and JD designed the search strategies and searched the literature. RT, GS, YB, and PS analyzed the data. RT, JD, XZ, and JZ drafted the manuscript. RT and JY selected the studies and abstracted the data. PS and $\mathrm{HZ}$ revised the manuscript. All authors read and approved the final manuscript.

\section{Funding}

This work was supported by National Clinical Research Center for Geriatrics, West China Hospital, Sichuan University (Z2018A01), Natural Science Foundation of China (NSFC 81672547), and 1.3.5 project for disciplines of excellence, West China Hospital, Sichuan University (No.0040205301E21).

\section{Availability of data and materials}

All data generated or analyzed during this study are included in this manuscript. 
Ethics approval and consent to participate

Not applicable.

\section{Consent for publication}

Not applicable.

\section{Competing interests}

The authors declare that they have no competing interests.

Received: 8 August 2019 Accepted: 23 September 2019

Published online: 09 November 2019

\section{References}

1. Sanda MG, Chen RC, Crispino T, Freedland S, Nelson M. Reston J, et al. Clinically localized prostate cancer: AUA/ASTRO/SUO guideline. 2017;6:27.

2. Stephenson AJ, Bolla M, Briganti A, Cozzarini C, Moul JW, Roach M 3rd, et al. Postoperative radiation therapy for pathologically advanced prostate cancer after radical prostatectomy. Eur Urol. 2012;61(3):443-51.

3. Stephenson AJ, Bolla M, Briganti A, Cozzarini C, Moul JW, Roach lii M, et al. Postoperative radiation therapy for pathologically advanced prostate Cancer after radical prostatectomy. Eur Urol. 2012;61(3):443-51.

4. Thompson IM, Valicenti RK, Albertsen P, Davis BJ, Goldenberg SL, Hahn C, et al. Adjuvant and salvage radiotherapy after prostatectomy: AUA/ASTRO guideline. J Urol. 2013;190(2):441-9.

5. Fossati N, Karnes RJ, Boorjian SA, Moschini M, Morlacco A, Bossi A, et al. Long-term impact of adjuvant versus early salvage radiation therapy in pT3NO prostate Cancer patients treated with radical prostatectomy: results from a multi-institutional series. Eur Urol. 2017;71(6):886-93.

6. Bolla M, van Poppel H, Collette L, van Cangh P, Vekemans K, Da Pozzo L, et al. Postoperative radiotherapy after radical prostatectomy: a randomised controlled trial (EORTC trial 22911). Lancet. 2005;366(9485):572-8.

7. Thompson IM, Tangen CM, Paradelo J, Lucia MS, Miller G, Troyer D, et al. Adjuvant radiotherapy for pathological T3NOMO prostate cancer significantly reduces risk of metastases and improves survival: long-term followup of a randomized clinical trial. J Urol. 2009;181(3):956-62.

8. Wiegel T, Bottke D, Steiner U, Siegmann A, Golz R, Storkel S, et al. Phase III postoperative adjuvant radiotherapy after radical prostatectomy compared with radical prostatectomy alone in pT3 prostate cancer with postoperative undetectable prostate-specific antigen: ARO 96-02/AUO AP 09/95. I Clin Oncol. 2009;27(18):2924-30.

9. Sineshaw HM, Gray PJ, Efstathiou JA, Jemal A. Declining use of radiotherapy for adverse features after radical prostatectomy: results from the National Cancer Data Base. Eur Urol. 2015;68(5):768-74.

10. Wells G, Shea B, O'connell D, Peterson J, Welch V, Losos M, et al. The Newcastle-ottawa Quality Assessment Scale (NOS) for assessing the quality of nonrandomised studies in meta-analyses. 2017. http://www.ohri.ca/ programs/clinical_epidemiology/oxford.asp of subordinate document.

11. Anjewierden S, Han Z, Foster CB, Pant C, Deshpande A. Risk factors for Clostridium difficile infection in pediatric inpatients: a meta-analysis and systematic review. Infect Control Hosp Epidemiol. 2019;40(4):420-6.

12. Tierney JF, Stewart LA, Ghersi D, Burdett S, Sydes MR. Practical methods for incorporating summary time-to-event data into meta-analysis. Trials. 2007;8:16.

13. Sun $\mathrm{P}$, Yang $\mathrm{X}, \mathrm{He} \mathrm{RQ}, \mathrm{Hu} \mathrm{QG}$, Song ZF, Xiong J, et al. Antiviral therapy after curative treatment of hepatitis $B / C$ virus-related hepatocellular carcinoma: a systematic review of randomized trials. Hepatol Res. 2014;44(3):259-69.

14. Briganti A, Wiegel T, Joniau S, Cozzarini C, Bianchi M, Sun M, et al. Early salvage radiation therapy does not compromise cancer control in patients with pT3NO prostate cancer after radical prostatectomy: results of a match-controlled multi-institutional analysis. Eur Urol. 2012;62(3):472-87.

15. Buscariollo DL, Drumm M, Niemierko A, Clayman RH, Galland-Girodet S, Rodin D, et al. Long-term results of adjuvant versus early salvage postprostatectomy radiation: a large single-institutional experience. Pract Radiat Oncol. 2017;7(2):e125-e33.

16. Fossati N, Karnes RJ, Boorjian SA, Moschini M, Morlacco A, Bossi A, et al. Long-term impact of adjuvant versus early salvage radiation therapy in PT3NO prostate Cancer patients treated with radical prostatectomy: results from a multi-institutional series. Eur Urol. 2017;71(6):886-93.

17. Hwang WL, Tendulkar RD, Niemierko A, Agrawal S, Stephans KL, Spratt DE, et al. Comparison between adjuvant and early-salvage Postprostatectomy radiotherapy for prostate Cancer with adverse pathological features. JAMA Oncol. 2018;4(5):e175230.
18. Borghetti P, Spiazzi L, Cozzaglio C, Pedretti S, Caraffini B, Triggiani L, et al. Postoperative radiotherapy for prostate cancer: the sooner the better and potential to reduce toxicity even further. La radiologia medica. 2017;123(1):63-70.

19. Hervas A, Gomez-Caamano A, Casana M, Gomez-Iturriaga A, Pastor J, Jove J, et al. Adjuvant versus salvage radiotherapy in prostate cancer: multiinstitutional retrospective analysis of the Spanish RECAP database. Clin Transl Oncol. 2018;20(2):193-200.

20. Mishra MV, Scher ED, Andrel J, Margules AC, Hegarty SE, Trabulsi EJ, et al. Adjuvant versus salvage radiation therapy for prostate Cancer patients with adverse pathologic features. Am J Clin Oncol. 2015;38(1):55-60.

21. Hsu CC, Paciorek AT, Cooperberg MR, Roach M, Hsu ICJ, Carroll PR. Postoperative radiation therapy for patients at high-risk of recurrence after radical prostatectomy: does timing matter? BJU Int. 2015;116(5):713-20.

22. Tilki $D$, Preisser F, Tennstedt $P$, Tober $P$, Mandel $P$, Schlomm T, et al. Adjuvant radiation therapy is associated with better oncological outcome compared with salvage radiation therapy in patients with $\mathrm{pN} 1$ prostate cancer treated with radical prostatectomy. BJU Int. 2017;119(5):717-23.

23. Detti B, Scoccianti S, Cassani S, Cipressi S, Villari D, Lapini A, et al. Adjuvant and salvage radiotherapy after prostatectomy: outcome analysis of 307 patients with prostate cancer. J Cancer Res Clin Oncol. 2012;139(1):147-57.

24. Ost P, De Troyer B, Fonteyne V, Oosterlinck W, De Meerleer G. A matched control analysis of adjuvant and salvage high-dose postoperative intensitymodulated radiotherapy for prostate Cancer. International Journal of Radiation Oncology Biology Physics. 2011;80(5):1316-22.

25. Trabulsi EJ, Valicenti RK, Hanlon AL, Pisansky TM, Sandler HM, Kuban DA, et al. A multi-institutional matched-control analysis of adjuvant and salvage postoperative radiation therapy for pT3-4NO prostate Cancer. Urology. 2008; 72(6):1298-302.

26. Tsien C, Griffith KA, Sandler HM, McLaughlin P, Sanda MG, Montie J, et al. Long-term results of three-dimensional conformal adjuvant and salvage radiotherapy after radical prostatectomy. Urology. 2003;62(1):93-8.

27. Taylor N, Kelly JF, Kuban DA, Babaian RJ, Pisters LL, Pollack A. Adjuvant and salvage radiotherapy after radical prostatectomy for prostate cancer. International Journal of Radiation Oncology Biology Physics. 2003;56(3):755-63.

28. Kalapurakal JA, Huang C-F, Neriamparampil MM, Small WJ, Pins MR, Mittal $\mathrm{BB}$, et al. Biochemical disease-free survival following adjuvant and salvage irradiation after radical prostatectomy. International Journal of Radiation Oncology Biology Physics. 2002;54(4):1047-54.

29. Morris MM, Dallow KC, Zietman AL, Park J, Althausen A, Heney NM, et al. Adjuvant and salvage irradiation following radical prostatectomy for prostate cancer. International Journal of Radiation Oncology Biology Physics. 1997:38(4):731-6.

30. Do LV, Do TM, Smith R, Parker RG. Postoperative radiotherapy for carcinoma of the prostate: impact on both local control and distant disease-free survival. American Journal of Clinical Oncology: Cancer Clinical Trials. 2002;25(1):1-8.

31. Jereczek-Fossa BA, Zerini D, Vavassori A, Fodor C, Santoro L, Minissale A, et al. Sooner or later? Outcome analysis of 431 prostate cancer patients treated with postoperative or salvage radiotherapy. International Journal of Radiation Oncology Biology Physics. 2009;74(1):115-25.

32. Pacholke HD, Wajsman Z, Algood CB, Morris CG, Zlotecki RA. Postoperative adjuvant and salvage radiotherapy for prostate cancer: impact on freedom from biochemical relapse and survival. Urology. 2004;64(5):982-6.

33. Wadasaki K, Kaneyasu Y, Kenjo M, Matsuura K, Murakami Y, Hashimoto Y, et al. Treatment results of adjuvant radiotherapy and salvage radiotherapy after radical prostatectomy for prostate cancer. Int J Clin Oncol. 2007;12(1):37-41.

34. Wiegel T, Bartkowiak D, Bottke D, Bronner C, Steiner U, Siegmann A, et al. Adjuvant radiotherapy versus wait-and-see after radical prostatectomy: 10-year follow-up of the ARO 96-02/AUO AP 09/95 trial. Eur Urol. 2014;66(2):243-50.

35. Bolla M, van Poppel H, Tombal B, Vekemans K, Da Pozzo L, de Reijke TM, et al. Postoperative radiotherapy after radical prostatectomy for high-risk prostate cancer: long-term results of a randomised controlled trial (EORTC trial 22911). Lancet. 2012;380(9858):2018-27.

36. Catton C. Post-operative radiotherapy following radical prostatectomy. EAU Updat Ser. 2005;3(2):107-16.

37. Thompson IM, Valicenti RK, Albertsen P, Davis BJ, Goldenberg SL, Hahn C, et al. Adjuvant and salvage radiotherapy after prostatectomy: AUA/ASTRO guideline. J Urol. 2013;190(2):441-9.

38. van Oort IM, Hulsbergen-vandeKaa CA, Witjes JA. Prognostic factors in radical prostatectomy specimens: what Do we need to know from pathologists? Eur Urol Suppl. 2008;7(12):715-22. 
39. Swanson GP, Basler JW. Prognostic factors for failure after prostatectomy. J Cancer. 2011;2:1-19.

40. Pearse M, Fraser-Browne C, Davis ID, Duchesne GM, Fisher R, Frydenberg M, et al. A phase III trial to investigate the timing of radiotherapy for prostate cancer with high-risk features: background and rationale of the radiotherapy -- adjuvant versus early salvage (RAVES) trial. BJU Int. 2014;113(Suppl 2):7-12.

\section{Publisher's Note}

Springer Nature remains neutral with regard to jurisdictional claims in published maps and institutional affiliations.

Ready to submit your research? Choose BMC and benefit from:

- fast, convenient online submission

- thorough peer review by experienced researchers in your field

- rapid publication on acceptance

- support for research data, including large and complex data types

- gold Open Access which fosters wider collaboration and increased citations

- maximum visibility for your research: over $100 \mathrm{M}$ website views per year

At $\mathrm{BMC}$, research is always in progress.

Learn more biomedcentral.com/submissions 